\title{
QUINOLINAS: PROMISSORES SCAFFOLDS PARA FUTUROS AGENTES ANTIINFLAMATÓRIOS
}

\author{
S. E. B. SILVA ${ }^{1}$, M. L. ALMEIDA ${ }^{2}$, I. R. PITTA ${ }^{2}$, M. G. R. PITTA ${ }^{2}$ \\ 1 Laboratório de Planejamento e Síntese de Fármacos (LPSF); Laboratório de \\ Imunopatologia Keizo Asami (LIKA), Universidade Federal de Pernambuco (UFPE) \\ ${ }^{2}$ Laboratório de Planejamento e Síntese de Fármacos (LPSF), Universidade Federal de \\ Pernambuco (UFPE)
}

RESUMO - O anel quinolínico é responsável por diversas atividades farmacológicas. Dentre elas, ele possui ação anti-inflamatória. Os AINEs são extensamente utilizados no combate do processo inflamatório que acomete algumas doenças. Atualmente há várias pesquisas que avaliam derivados quinolínicos com as mais variadas funções orgânicas ligadas ao anel, além de grupos aminas e átomos eletronegativos que fazem dessas moléculas interessantes candidatos a fármacos. O objetivo desse trabalho é reunir os artigos mais recentes e elencar as características das moléculas observando suas similaridades, diferenças e parâmetros relacionados a regra dos cinco de Lipinski. Dentre os 26 trabalhos apresentados, muitos compostos se enquadram na regra e sobressaem as similaridades entre eles. Nesta revisão, consta as moléculas mais ativas de artigos científicos em um periodo de 10 anos (2007-2017), e pode servir de base para o planejamento de compostos potentes, seguros e eficazes, e com menos chances de serem eliminados nas diferentes fases da cadeia produtiva de fármacos.

Palavras-chave: anel quinolínico, ação anti-inflamatória, AINEs

ABSTRACT - The quinoline ring is responsible for several pharmacological activity. Among them, it has anti-inflammatory action. NSAIDs are widely used in the fight against the inflammatory process that affects some diseases. There are several studies that evaluate quinoline derivatives with the most varied organic functions linked to the ring, as well as amine groups and electronegative atoms that make these molecules interesting drug candidates. The objective of this work is to gather the most recent articles and list the characteristics of the molecules observing their similarities, differences and parameters related to the Rule of the five of Lipinski. Among the 26 papers presented, many compounds fall under the rule and the similarities between them stand out. In this review we can find the most active molecules of scientific articles in a period of 10 years (2007-2017), and can serve as a basis for the planning of potent, safe and effective compounds, and less likely to be eliminated in the different phases of the chain production of drugs.

Keywords: quinoline ring, anti-inflammatory action, NSAIDs 


\section{INTRODUÇÃO}

"Inflamação, a primeira resposta do sistema imune ao estímulo prejudicial como uma infecção ou irritação, consistindo de uma cascata de eventos bioquímicos que propaga e matura a resposta inflamatória" (MUKHERJEE, 2013). Com outras palavras, o processo inflamatório além de ser uma defesa do organismo, serve de alerta para algo prejudicial ao mesmo. Além disso, este processo está presente em algumas doenças como: artrite reumatoide, inflamação intestinal aguda, doenças inflamatórias da pele, dentre outras, vinculadas a uma resposta inflamatória exacerbada (NETEA, 2017).

Os principais agentes da resposta inflamatória são os mediadores da inflamação histamina, eicosanoides, citocinas, bradicinina. Os Anti-inflamatórios não-esteroidais (AINEs), classe de medicamentos, atuam no combate da inflamação impedindo a formação dos eicosanoides. Sua síntese inicia-se pela atuação da fosfolipase $\mathrm{A}_{2}$ nos fosfolipídeos para produção do araquidonato, o qual sofrerá ações das enzimas ciclooxigenase (COX) e lipoxigenase, que produzirão prostaglandinas, tromboxanos e leucotrienos (eicosanoides). Dentre as ações desses mediadores podemos citar a vasodilatação, que implica no aumento do fluxo sanguíneo e eritema no local da inflamação (RANG, 2004).

O mecanismo de ação dos AINES compreende a inibição das COXs, porém, seu uso necessita de limitação e cautela por promover efeitos colaterais gastrointestinais. A COX é uma proteína presente na membrana que possui três isoformas, COX-1 (mantém a homeostasia), COX-2 (induz a inflamação) (ZARGHI, 2009) e COX-3 (pouco conhecida). Os inibidores seletivos COX-2, Coxibs, foram uma descoberta promissora devido sua seletividade, entretanto, observou-se efeitos adversos cardiovasculares, o que resultou na retirada do mercado de alguns medicamentos dessa classe como, Refocoxib (Vioxx) (ADBELRAHMAN, 2016).

Em meio a necessidade atual da Química Medicinal da alta seletividade e pouco/nenhum efeito adverso, grupos farmacofóricos estão sendo explorados minuciosamente no intuito de encontrar um derivado que possua ação anti-inflamatória com características promissoras. A quinolina ou 1-aza-naftaleno (Fig. 1) é a estrutura base para muitas moléculas com propriedades farmacológicas diferentes, presente em vários fármacos como, clioquinol (infecções fúngicas cutâneas), montelucaste de sódio (asma crônica e renite alérgica), brequinar sódico (imunossupressor), amodiaquina (antimalárico), floctafenina (AINE), dentre outras (PINHEIRO, 2010). Além destes, existem vários artigos que comprovam o efeito anti-inflamatório dos derivados quinolínicos (MORELLA, 2013).

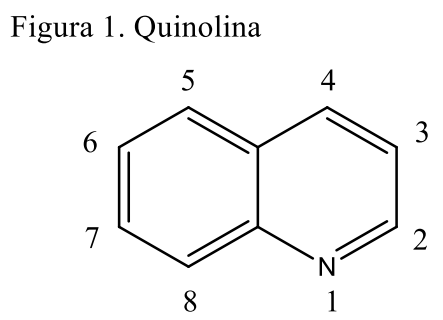

Diante da necessidade de encontrar novas e mais ativas moléculas com ação antiinflamatória, capazes de combater doenças envolvidas com processo inflamatório, investir em derivados quinolínicos pode ser promissor, visto que esses derivados abarcam um amplo espectro farmacológico. Por esse motivo, realizou-se este levantamento bibliográfico que poderá ajudar pesquisas futuras no planejamento de uma série de compostos fundamentados em características mais relevantes apresentadas nos artigos aqui analisados. 


\section{MATERIAIS E MÉTODOS}

Para o presente trabalho foi utilizado a plataforma PeriódicosCapes pelo acesso a partir da UFPE. Neste, explorou-se os bancos de dados PubMed e SciFinder fazendo uma busca com as seguintes palavras-chave: quinoline derivatives and antiinflammatory activity, no período de 27/10/2017 a 04/11/2017, limitando-se aos artigos presentes na última década (2007-2017).

Esta pesquisa resultou na obtenção de 205 artigos, os quais podiam conter todas as palavras ou aqueles que continham uma das duas opções, sendo necessário fazer uma seleção minuciosa para separar os artigos que contemplavam os derivados quinolínicos com atividade anti-inflamatória.

\section{RESULTADOS E DISCUSSÃO}

Dentre os 205 artigos encontrados, apenas 26 deles abarcam o parâmetro de inclusão. Desses, 9 artigos foram selecionados pela base SciFinder e 17 pelo PubMed. Na tabela 1, pode ser vista as principais moléculas e seus respectivos nomes químicos citados nos artigos.

Os alvos biológicos, em sua maioria, compreendem as ciclooxigenases, o que sugere classificar essas moléculas no grupo de anti-inflamatórios não-esteroidais. Quanto o alvo COX-2 das moléculas 20 e 18, provavelmente se deve pela presença do grupo metilsulfonil ligado ao benzeno, o que comprova sua similaridade com a molécula de celecoxib, a qual possui uma sulfonamida ligada ao benzeno, sendo esses substituintes importantes para a seletividade pela COX-2 (GHODSI, 2010).

Quanto aos substituintes mais presentes, os nitrogenados aparecem na maioria das moléculas selecionadas, muitos dos quais, compreendem os azoles (moléculas 3, 19, 9), mas também pirimido (moléculas 22, 24), oxazina (molécula 10), azetidinona (molécula 7), ureia (molécula 13). A presença do nitrogênio é também observada nos fármacos antiinflamatórios: indometacina, diclofenaco, celecoxib, utilizados como droga padrão nos ensaios de atividade dos compostos, o que se pode inferir que a presença de nitrogênio nessas moléculas deve contribuir para a atividade farmacológica.

A ação farmacológica de uma molécula está relacionada a promissoras características de solubilidade e permeabilidade. Segundo Lipinski (1997), para que uma molécula tenha tais características devem seguir 4 parâmetros fundamentais, conhecida como Regra dos cinco: possuir quantidade de doadores de $\mathrm{H}<5$, de receptor de $\mathrm{H}<10$, peso molecular $<500$ e clogP $<5$. Dos 26 compostos apresentados, seis, não obedecem a essa regra. As moléculas 4,12,15, 19, 21 e 26 possuem peso molecular $>500$ e clogP $>5$, exceto a molécula 21 , que possui cLogP 3,7 , pois nesta, está ligada uma molécula de galactose, a qual possui cinco hidroxilas ao longo da cadeia carbônica, conferindo uma certa hidrofilidade a molécula. $\mathrm{O}$ cLogP está relacionado a afinidade da molécula pela fase orgânica ou aquosa, valores muito altos implica numa alta solubilidade pela fase orgânica.

Entretanto, para uma molécula ser bem absorvida, não basta conhecer apenas sua solubilidade, o peso molecular que está relacionado ao tamanho da molécula, atribuirá uma facilidade ou não desta se difundir nas células, além da existência de átomos que possuem a propriedade de aceitar ou doar hidrogênio do meio, poderá comprometer a permeabilidade da molécula.

Embora todas as moléculas relacionadas na tabela 1 tenham apresentado atividade anti-inflamatória, seus parâmetros ADMET (absorção, distribuição, metabolismo, eliminação e toxicidade) devem ser avaliados, e a regra de Lipinski pode ser útil na 
seleção de quais moléculas possuem melhor predição e que valha a pena fazer novas avaliações, ou até mesmo, a partir dessa informação, tentar reproduzir características químicas marcantes e funcionais para elaboração de novos grupos de moléculas.

Tabela 1 - Principais moléculas de derivados quinolínicos com atividade antiinflamatória, classificadas pelo ano de publicação.

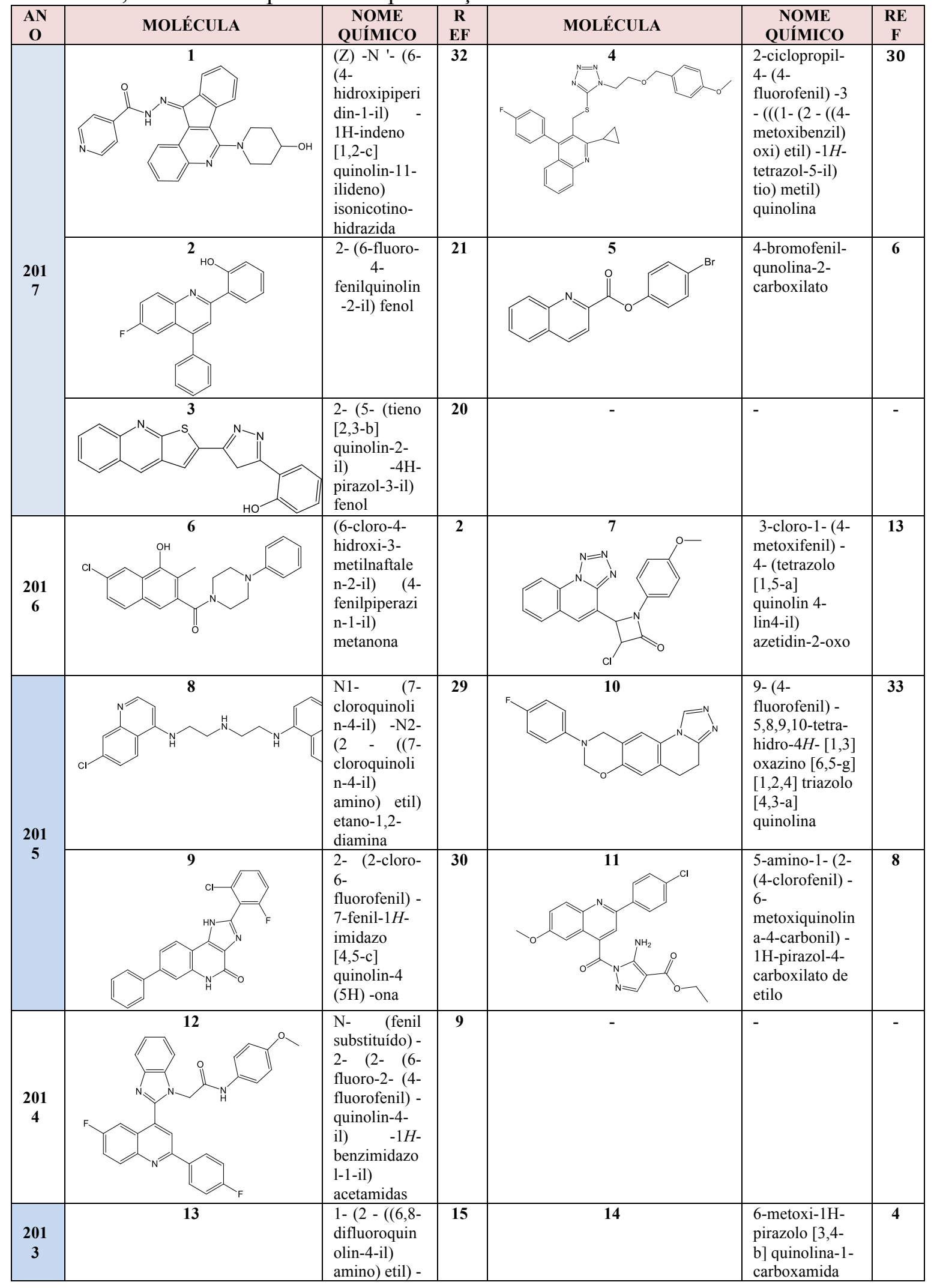




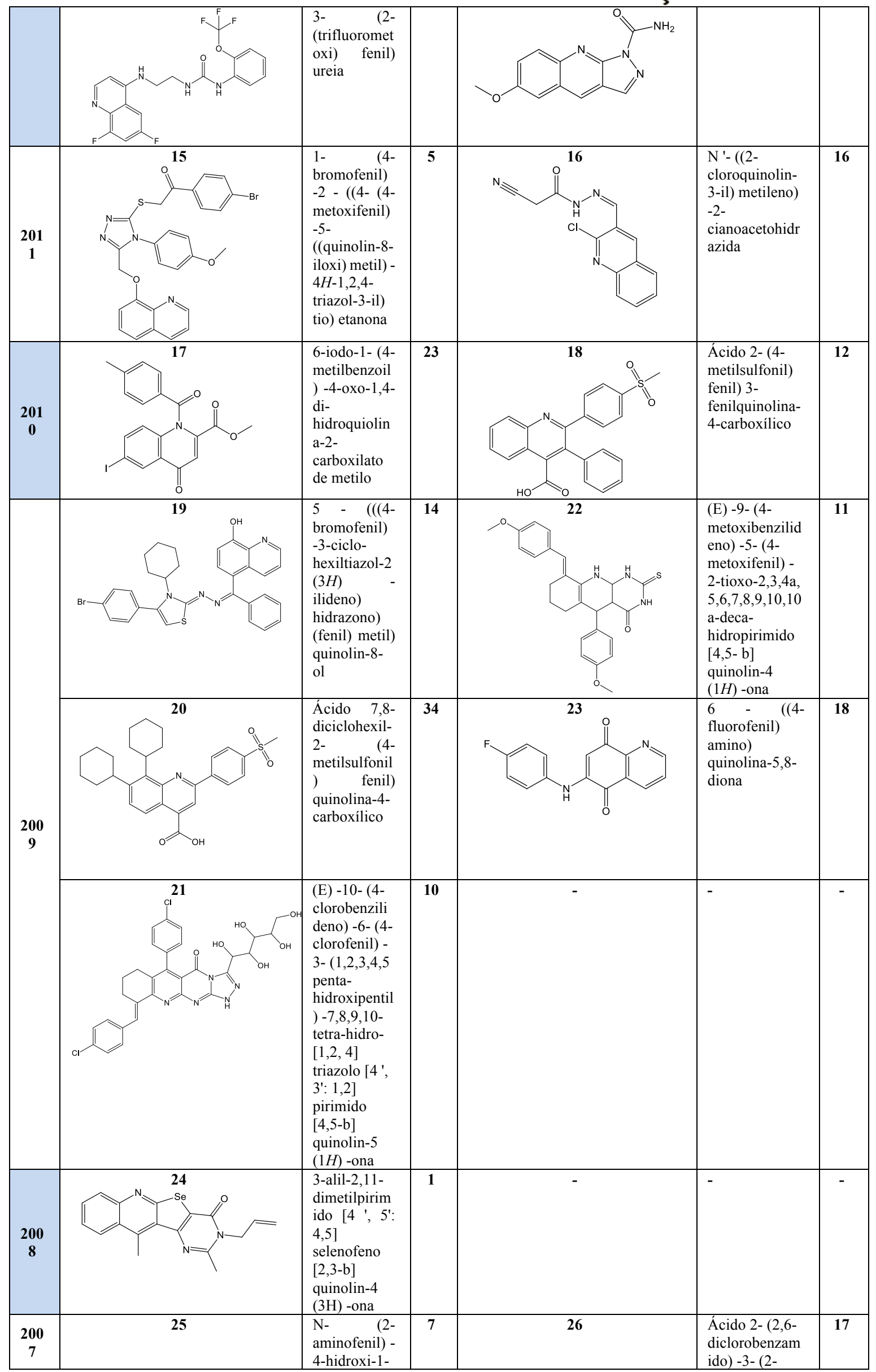




\section{CONCLUSÃO}

O anel quinolínico chama a atenção da comunidade científica pela sua capacidade de formar derivados com as mais variadas atividades farmacológicas. Neste trabalho, selecionaram-se apenas artigos que possuíam compostos com atividade antiinflamatória.

Muitos desses compostos agregam características interessantes - peso molecular $<500, \operatorname{cLogP}<5$, substituintes nitrogenados e funções orgânicas promissoras fazem dessas moléculas candidatas a fármacos. Além disso, sua capacidade de inibir, principalmente, enzimas responsáveis pela síntese de mediadores da inflamação, direciona a pesquisa para avaliar sua resposta frente a situações que mimetizam o microambiente de algumas doenças.

Várias enfermidades como a artrite reumatoide, inflamação intestinal aguda, doenças inflamatórias da pele, são acometidas por um processo inflamatório, que requerem novos compostos que possam eliminar as inconveniências da inflamação e agregar qualidade de vida a população.

A importância de reunir vários trabalhos e mostrar suas moléculas mais potentes e seus alvos biológicos pode facilitar no planejamento de novos derivados quinolínicos com melhor atividade e parâmetros ADMET que permitam que esses novos compostos sejam promissores candidatos a fármacos.

\section{REFERÊNCIAS}

1. ABDEL-HAFEZ, S. H., HUSSEN, M. A. Selenium-containing heterocycles: synthesis and pharmacological activities of some new 4-methylquinoline-2 $(1 \mathrm{H})$ selenone derivatives. Arch Pharm (Weinheim), 341(4), p 240-6., 2008.

2. ABDElrahman, M. H., YOUSSIF, B. G. M., ABDElGAWAD, M. A. et al. Synthesis, biological evaluation, docking study and ulcerogenicity profiling of some novel quinoline-2-carboxamides as dual COXs/LOX inhibitors endowed with antiinflammatory activity . European Journal of Medicinal Chemistry, p 1-14, 2016.

3. ADBELRAHMAN, M. H., YOUSSIF, B. G. M., ABDELGAWAD, M. A., et al. Synthesis, biological evaluation, docking study and ulcerogenicity profiling of some novel quinoline-2-carboxamides as dual COXs/LOX inhibitors endowed with antiinflammatory activity. European Journal of Medicinal Chemistry , p 1-14, 2016.

4. AlAM, M. M., MORELLA, A., AKHTAR, M. et al. Microwave assisted one pot synthesis of some pyrazole derivatives as a safer anti-inflammatoryand analgesic agents. Acta Pol Pharm, 70(3), p 435-41, 2013.

5. ALAM, M. M., SHAHARYAR, M., HAMID, H. et al. Synthesis of novel 8-hydroxy quinolin based 1,3,4-oxadiazoles and S-substituted 1,2,4-triazole derivatives and 
evaluation of their anti-inflammatory, analgesic, ulcerogenic and anti-microbial activities. Med Chem, 7(6), p 663-73, 2011.

6. BOYARSHINOV, V.D., MIKHALEV, A. I., YUSHKOVA, T. A., UKHOV, S. V., KON'SHINA T. M. Synthesis and biological activity of quinoline-2-carboxylic acid aryl esters and amides. Pharmaceutical Chemistry Journal, 51(5), p. 25 - 28, 2017.

7. DETSI, A., BOULOUMBASI, D., PROUSIS, K. C. et al. Design and synthesis of novel quinolinone-3-aminoamides and their alpha-lipoic acid adducts as antioxidant and anti-inflammatory agents. J Med Chem, 50(10), p. 2450-8, 2007.

8. EL-FEKY, S. A. H., EL-SAMII, Z. K. A., OSMAN, N. A., LASHINE, J., KAMEL, M. A., THABET, H. K. Synthesis, molecular docking and anti-inflammatory screening of novel quinoline incorporated pyrazole derivatives using the Pfitzinger reaction II. Bioorganic Chemistry, 58, p. 104-116, 2015.

9. EL-FEKY, S. A., THABET, H. K., UBEID, M. T. Synthesis, molecular modeling and anti-inflammatory screening of novel fluorinated quinoline incorporated benzimidazole derivatives using the Pfitzinger reaction. Journal of Fluorine Chemistry, 161, p. 87-94, 2014.

10. EL-GAZZAR, A. B. A., HAFEZ, H. N., NAWWAR, G. A. M. New acyclic nucleosides analogues as potential analgesic, anti-inflammatory, anti-oxidant and antimicrobial derived from pyrimido[4,5-b]quinolines. Eur J Med Chem, 44(4), p 142736, 2009.

11. EL-GAZZAR, A. B. A., YOUSSEF, M. M., YOUSSEF, A. M. S. et al. Design and synthesis of azolopyrimidoquinolines, pyrimidoquinazolines as anti-oxidant, antiinflammatory and analgesic activities. Eur J Med Chem, 44(2), p 609-24, 2009.

12. GHODSI, R., ZARGHI A., DARAEI, B., HEDAYATI, M. Design, synthesis and biological evaluation of new 2,3-diarylquinoline derivatives as selective cyclooxygenase-2 inhibitors. Bioorg Med Chem, 18(3), p 1029-33, 2010.

13. GUPTA S.K., MISHRA, A. Synthesis, Characterization \& Screening for Anti Inflammatory \& Analgesic Activity of Quinoline Derivatives Bearing Azetidinones Scaffolds. Antiinflamm Antiallergy Agents Med Chem. 2016;15(1):31-43.

14. HUSSEIN, M. A., KAFAFY, A. N., ABDEL-MOTY, S. G., ABOU-GHADIR, O. M. Synthesis and biological activities of new substituted thiazoline-quinoline derivatives. Acta Pharm, 59(4), p 365-82, 2009.

15. KECHE, A. P., HATNAPURE, G. D., TALE, R. H., RODGE, A. H., BIRAJDAR, S. S., KAMBLE, V. M. Synthesis, anti-inflammatory and antimicrobial evaluation of novel N1-(quinolin-4yl)ethane-1,2-diamine phenyl urea derivatives. Med Chem Res, 22, p. 1480-1487, 2013.

16.KHIDRE, R. E., ABDEL-WAHAB, B. F., BADRIA, F. A. New Quinoline-Based Compounds for Analgesic and Anti-Inflammatory Evaluation. Letters in Drug Design \& Discovery, 8, p. 640-648, 2011.

17. LASSOIE, M., BROEDERS, F., COLLART, P. et al. 2,6-Quinolinyl derivatives as potent VLA-4 antagonists. Bioorg Med Chem Lett, 17(1):142-6, 2007.

18. LIM, K., LEE, J., LEE, S. et al. Potent anti-inflammatory effects of two quinolinedione compounds, OQ1 and OQ21, mediated by dual inhibition of inducible NO synthase and cyclooxygenase-2. Br J Pharmacol, 156(2), p 328-37, 2009.

19. LIPINSKI, C. A., LOMBARDO, F., DOMINY, B. W., FEENEY, P. J. Experimental and computational approaches to estimate solubility and permeability in drug discovery and development settings. Advanced Drug Delivery Reviews 23, p 3-25, 1997. 
20. MAHAJAN, P., NIKAM, M., ASRONDKAR, A., BOBADE, A., GILL, C. Synthesis, Antioxidant, and Anti-Inflammatory Evaluation of Novel Thiophene-Fused Quinoline Based $\beta$-Diketones and Derivatives. J. Heterocyclic Chem., 54, p.1415-1422, 2017.

21. MANIKANDAN, A., RAVICHANDRAN, S., SATHIYANATAYANAN, K. I., SIVAKUMAR, A. Efficacy of phenyl quinoline phenol derivatives as COX-2 inhibitors; an approach to emergent the small molecules as the anti-inflammatory and analgesic therapeutics. Inflammopharmacology, 2017.

22. MARELLA, A., TANWAR, O. P., SAHA, R. et al. Quinoline: a versatile heterocyclic. Saudi Pharmaceutical Journal, 21, p 1-12, 2013.

23. MAZZONI, O., ESPOSITO, G., DIURNO, M. V. et al. Synthesis and pharmacological evaluation of some 4-oxo-quinoline-2-carboxylic acid derivativesas antiinflammatory and analgesic agents. Arch Pharm (Weinheim), 343(10), p 561-9, 2010

24. MUKHERJEE, S.; PAL, M. Quinolines: a new hope against inflammation. Drug Discovery Today, v. 18, numbers 7/8, p 389-398, 2013.

25. NETEA, M. G.; BALKWILL, F.; CHONCHOL, M.; COMINELLI, F. et al. A guiding map for inflammation. Nature Immunology, v. 18 , number 8, 2017.

26. PINHEIRO,A.C., BISPO, M. L. F, SOUZA,M. V. N. A importância do núcleo quinolínico e seus derivados do desenvolvimento de fármacos. Revista Fitos, v. 5, 3, p 53-63, 2010.

27. PRACHAYASITTIKUL, V. et al. 8-Hydroxyquinolines: a review of their metal chelating properties and medicinal applications. Drug Des Devel Ther.,7, p 1157-78, 2013

28. RANG, H. P.; DALE, M. M.; RITTER, J. M. ; MOORE, P. K. Farmacologia. Rio de Janeiro: Editora Elsevier, $5^{\text {a }}$ edição, 2004.

29. SANTOS, R. M., BARROS, P. R., BORTOLUZZI, J. H. et al. Synthesis and evaluation of the anti-nociceptive and anti-inflammatory activity of 4aminoquinoline derivatives. Bioorg Med Chem, 23(15), p 4390-6, 2015.

30. SHIRO, T., FUKAYA, T., TOBE, M. The chemistry and biological activity of heterocycle-fused quinolinone derivatives: A review. Eur J Med Chem. ,97, p $397-$ 408, 2015.

31. SURESHKUMAR, K., MAHESHWARAN, V., RAO, T. D, THEMMILA, K., PONNUSWAMY, M. N., KADHIRVEL, S., DHANDAYUTHAM S. Synthesis, characterization, crystal structure, in-vitro antiinflammatory and molecular docking studies of 5-mercapto-1-substituted tetrazole incorporated quinoline derivative. Journal of Molecular Structure, 1146, p. 314-323, 2017.

32. TSENG, C., TUNG, C., WU, C. et al. Discovery of Indeno[1,2c]quinoline Derivatives as Potent Dual Antituberculosis and Anti-Inflammatory Agents. Molecules, 22, p 1-15, 2017.

33. WEN, X., WANG, S., LIU, D., GONG, G., QUAN, Z. Synthesis and evaluation of the anti-inflammatory activity of quinoline derivatives. Med Chem Res, 24, p. 25912603, 2015.

34. ZARGHI, A., GHODSI, R., AZIZI, E. et al. Synthesis and biological evaluation of new 4-carboxyl quinoline derivatives as cyclooxygenase-2 inhibitors. Bioorg Med Chem, 17(14), p 5312-7, 2009.

\section{AGRADECIMENTOS}

Agradeço ao acolhimento do Laboratório de Planejamento, Síntese de fármacos - LPSF, que faz parte do NUPIT SG e ao Laboratório de Imunopatologia Keizo Asami - LIKA por permitir contribuir com o conhecimento científico em prol da população. 